\title{
Assembled block copolymer stabilized high internal phase emulsion hydrogels for enhancing oil safety
}

\author{
Tao Zhang, ${ }^{\mathrm{a}}$ Zhiguang $\mathrm{Xu},{ }^{\mathrm{a}}$ Yuanpeng $\mathrm{Wu},{ }^{\mathrm{b}}$ and Qipeng Guo*a \\ a Polymers Research Group, Institute for Frontier Materials, Deakin University, Locked Bag \\ 20000, Geelong, Victoria 3220, Australia \\ ${ }^{\mathrm{b}}$ School of Materials Science and Engineering, Southwest Petroleum University, Chengdu, \\ Sichuan 610500, China \\ Corresponding author: E-mail: qguo@deakin.edu.au; Fax: +61 35227 1103; Tel: +61 35227 \\ 2802
}

Table of Contents

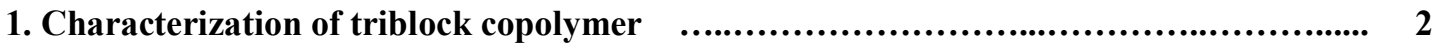

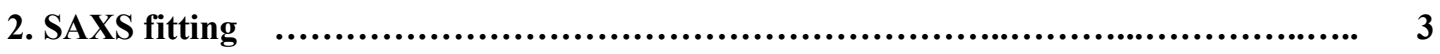

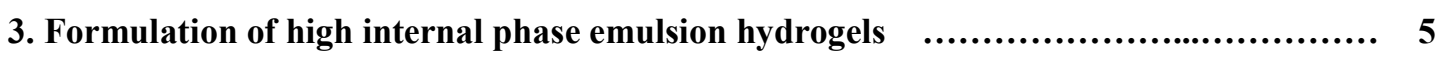

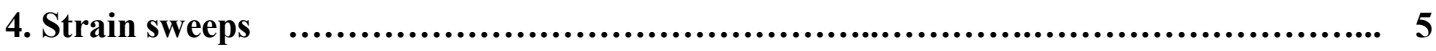




\section{Characterization of triblock copolymer}

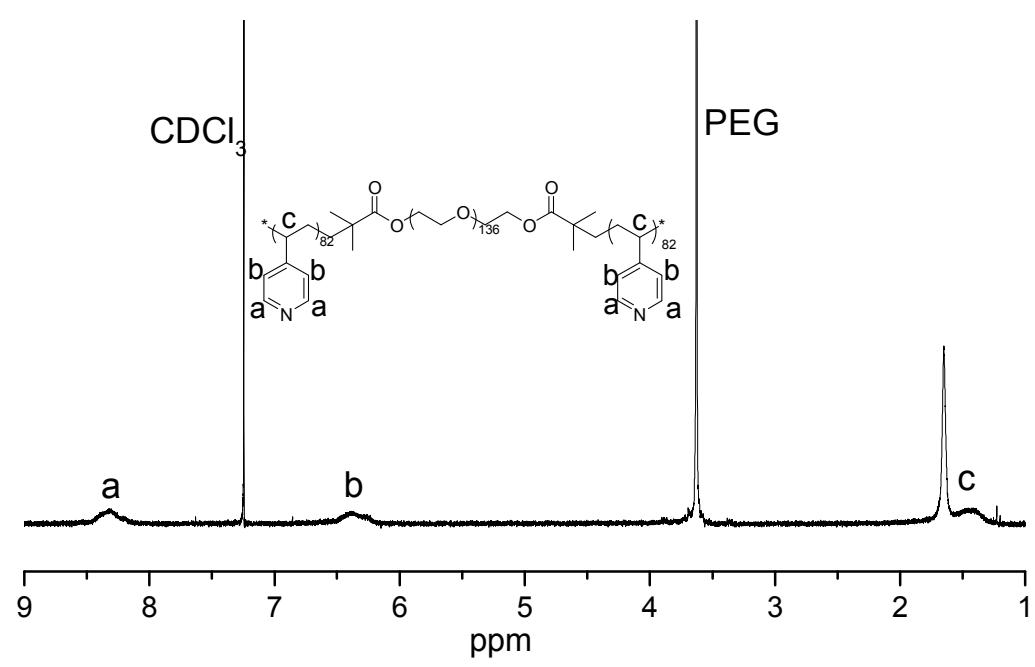

Figure $\mathrm{S} 1{ }^{1} \mathrm{H}-\mathrm{NMR}$ spectra of a typical $4 \mathrm{VP}_{\mathrm{m}}-\mathrm{EG}_{\mathrm{n}}-4 \mathrm{VP}_{\mathrm{m}}$ in $\mathrm{CDCl}_{3}$.

4VPm-EGn-4VPm was dissolved in $\mathrm{CDCl}_{3}$ to determine its chemical structure by ${ }^{1} \mathrm{H}$ NMR using a JEOL $270 \mathrm{MHz}$ NMR spectrometer. Figure S1 shows the ${ }^{1} \mathrm{H}-\mathrm{NMR}$ spectrum of a typical $4 \mathrm{VP}_{\mathrm{m}}-\mathrm{EG}_{\mathrm{n}}-4 \mathrm{VP}_{\mathrm{m}}$. The degree of polymerization (DP) for the $4 \mathrm{VP}$ block was calculated to be 82 from the ratio of the integrals of peaks $\mathbf{a}, \mathbf{b}$ and $\mathbf{c}$ to the peak of EG block. Taking into account the amount of protons in a EG monomer and groups $\mathbf{a}, \mathbf{b}$ and $\mathbf{c}$ of the $4 \mathrm{VP}$ block, the DP was determined by DP $=\frac{4\left(I_{a}+I_{b}+I_{c}\right)}{7 I_{P E G}} N_{E G}$ with $I_{a}, I_{b}$ and $I_{c}$ denote the integral of peaks $\mathbf{a}, \mathbf{b}$ and $\mathbf{c}$, respectively; $I_{E G}$ represents the integral of the EG peak and $N_{E G}=136$ the degree of polymerization of the starting PEG. Based on the results, the $4 \mathrm{VP}_{\mathrm{m}}-\mathrm{EG}_{\mathrm{n}}-4 \mathrm{VP}_{\mathrm{m}}$ is $4 \mathrm{VP}_{82}-\mathrm{EG}_{136}-4 \mathrm{VP}_{82}$.

Using the same methods, the structures of the other three $4 \mathrm{VP}_{\mathrm{m}}-\mathrm{EG}_{\mathrm{n}}-4 \mathrm{VP}_{\mathrm{m}}$ are calculated to be $4 \mathrm{VP}_{36}-\mathrm{EG}_{454}-4 \mathrm{VP}_{36}, 4 \mathrm{VP}_{87}-\mathrm{EG}_{227}-4 \mathrm{VP}_{87}$ and $4 \mathrm{VP}_{50}-\mathrm{EG}_{227}-4 \mathrm{VP}_{50}$.

Table S1 GPC results of $4 \mathrm{VP}_{\mathrm{m}}-\mathrm{EG}_{\mathrm{n}}-4 \mathrm{VP}_{\mathrm{m}}$

\begin{tabular}{cccc}
\hline & Chemicals & $\mathrm{M}_{\mathrm{n}}$ & PDI \\
\hline 1 & $4 \mathrm{VP}_{82}-\mathrm{EG}_{136}-4 \mathrm{VP}_{82}$ & 17,300 & 2.05 \\
2 & $4 \mathrm{VP}_{36}-\mathrm{EG}_{454}-4 \mathrm{VP}_{36}$ & 79,300 & 2.59 \\
3 & $4 \mathrm{VP}_{50}-\mathrm{EG}_{227}-4 \mathrm{VP}_{50}$ & 49,800 & 2.37 \\
4 & $4 \mathrm{VP}_{87}-\mathrm{EG}_{227}-4 \mathrm{VP}_{87}$ & 57,300 & 2.75 \\
\hline
\end{tabular}




\section{SAXS fitting}

The obtained SAXS scattering data were corrected for background scattering. The curve fittings were carried out using SASfit program, which was downloaded from http://kur.web.psi.ch/sans1/SANSSoft/sasfit.html. The middle EG block is totally dissoluble in water, and the end $4 \mathrm{VP}$ blocks may not dissolve into aqueous solution according to $\mathrm{pH}$ values. The $4 \mathrm{VP}$ blocks tend to form cores with EG block forming shell to stabilize the hydrophobic cores. The structures can be similar to star polymers which have all branches emanating from a core of the macromolecules. Therefore, a form factor of Dozier was considered and actually the form factor of Dozier 2 (re-parametrization of the Dozier) to scale the scattering of the star to the local scattering of the arms was used.

$$
I_{\text {Dozierstar } 2}\left(Q, I_{0}, R_{G}, v, \xi\right)=\frac{I_{0}}{N_{\text {agg }}}\left(\left(N_{\text {agg }}-1\right) \exp \left(-\frac{Q^{2} R_{G}^{2}}{3}\right)+\frac{\Gamma(\mu)}{Q \xi} \frac{\sin (\mu \arctan (Q \xi))}{\left(1+Q^{2} \xi^{2}\right)^{\mu / 2}}\right)
$$

With $\mu=1 / v-1$

$\mathrm{R}_{\mathrm{G}}$ : radius of gyration of the star

$\mathrm{I}_{0}$ : scale parameter

$\mathrm{N}_{\text {agg: }}$ number of arms in the star

$\xi$ : exponential damping length in mass fractal

$v$ : Flory exponent, $v=3 / 5$ in good solvent, $v=1 / 2$ in theta solvent (i.e. $v=2 / 3$ to 1 )

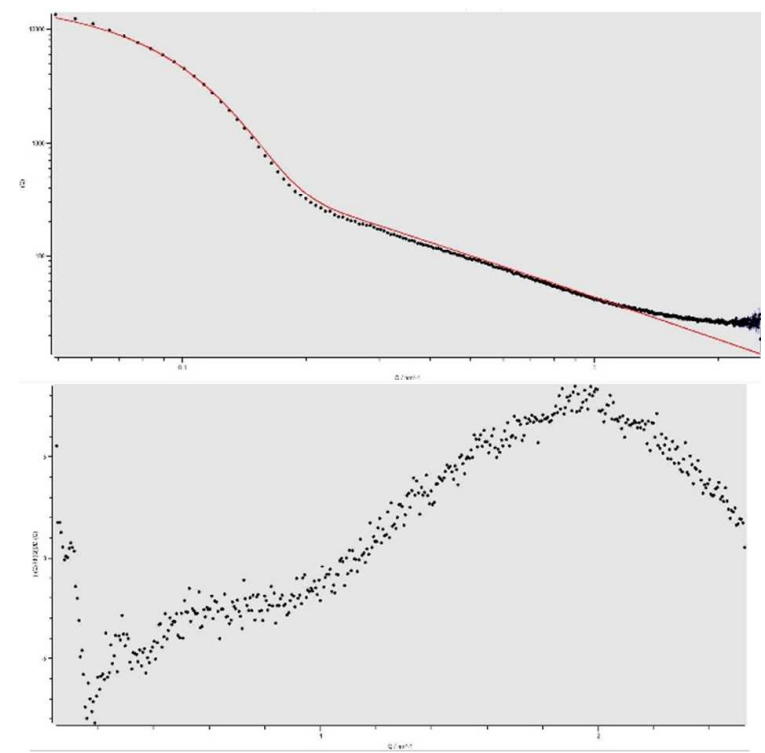

Figure S2 (a) SAXS fit of $4 \mathrm{VP}_{82}-\mathrm{EG}_{136}-4 \mathrm{VP}_{82}$ before dialysis (b) and uncertainties in the fit. Size distribution: Delta $N=36.3$. Form factor: DozierStar2; Parameters: $I_{0}=500 ; R_{G}=20.5 ; N_{a g g}=9 ; \xi=$ $27.5 ; v=0.8$. 

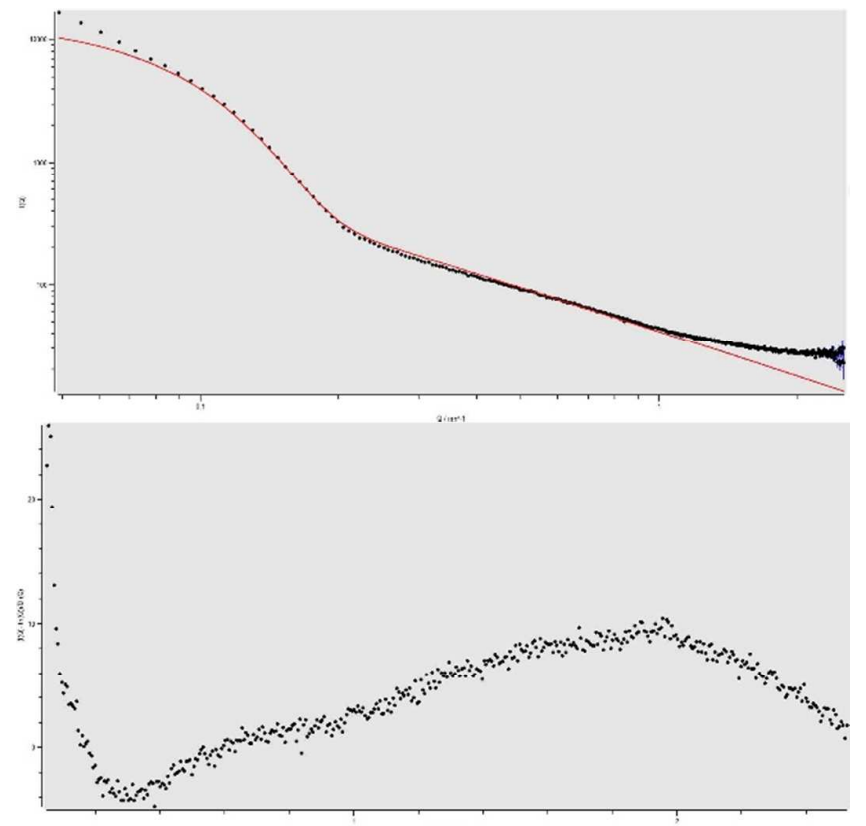

Figure S3 (a) SAXS fit of $4 \mathrm{VP}_{82}-\mathrm{EG}_{136}-4 \mathrm{VP}_{82}$ after dialysis (b) and uncertainties in the fit. Size distribution: Delta $N=29$. Form factor: DozierStar2; Parameters: $\mathrm{I}_{0}=500 ; \mathrm{R}_{\mathrm{G}}=20 ; \mathrm{N}_{\mathrm{agg}}=9 ; \xi=25$; $v=0.81$.

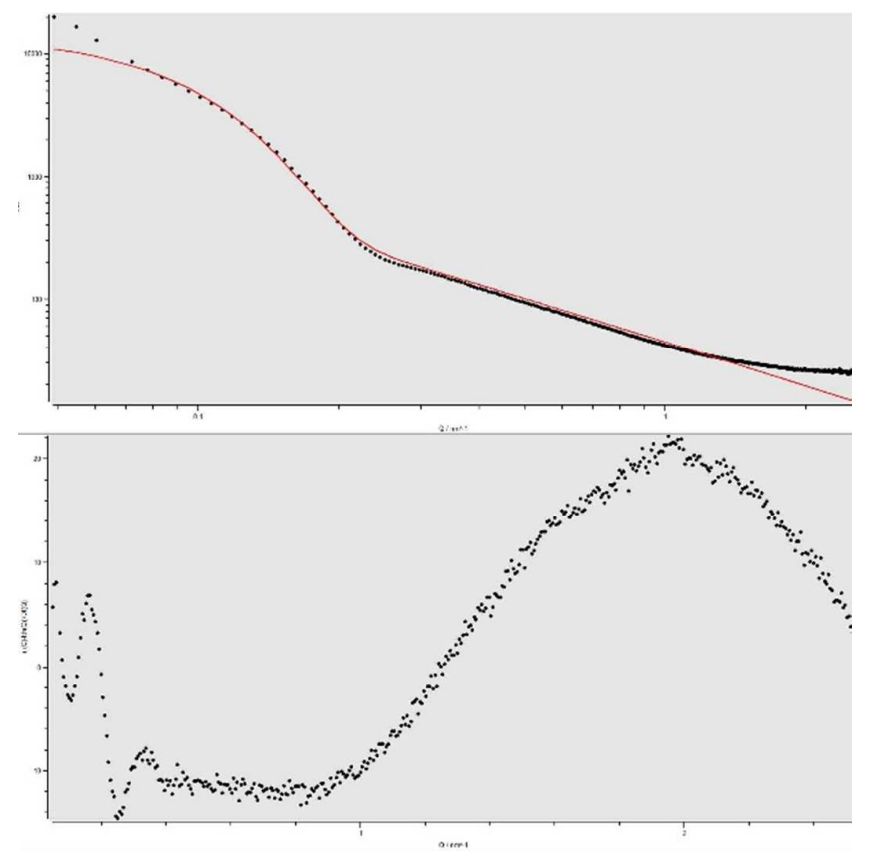

Figure S4 (a) SAXS fit of pH-driven assembled $4 \mathrm{VP}_{82}-\mathrm{EG}_{136}-4 \mathrm{VP}_{82}$ after (b) and uncertainties in the fit. Size distribution: Delta $N=27$. Form factor: DozierStar2; Parameters: $I_{0}=550 ; R_{G}=18.5 ; N_{a g g}=$ $9 ; \xi=25 ; v=0.82$. 


\section{Formulation of high internal phase emulsion hydrogels}

Table S2 Formulation of a typical HIPE hydrogels

\begin{tabular}{lll}
\hline & Components & Amounts \\
\hline Continuous & $4 \mathrm{VP}_{82}-\mathrm{EG}_{136}-4 \mathrm{VP}_{82}$ & $1 \%$ of the aqueous phase (w/v) \\
aqueous phase & Aqueous solution with $\mathrm{pH} 8$ & $20 \%$ of the total volume (v/v) \\
Dispersed organic & Toluene & $80 \%$ of the total volume \\
phase & &
\end{tabular}

Toluene in the dispersed organic phase can be changed into xylene, gasoline, diesel and engine oil. The volume fraction of the organic phase can be increase up to $89 \%$. The $4 \mathrm{VP}_{82}-\mathrm{EG}_{136}-4 \mathrm{VP}_{82}$ can be changed into other BCPs mentioned in manuscript, but the corresponding volume fraction of the organic phase may vary according to manuscript.

\section{Strain sweeps of a typical HIPE hydrogel}

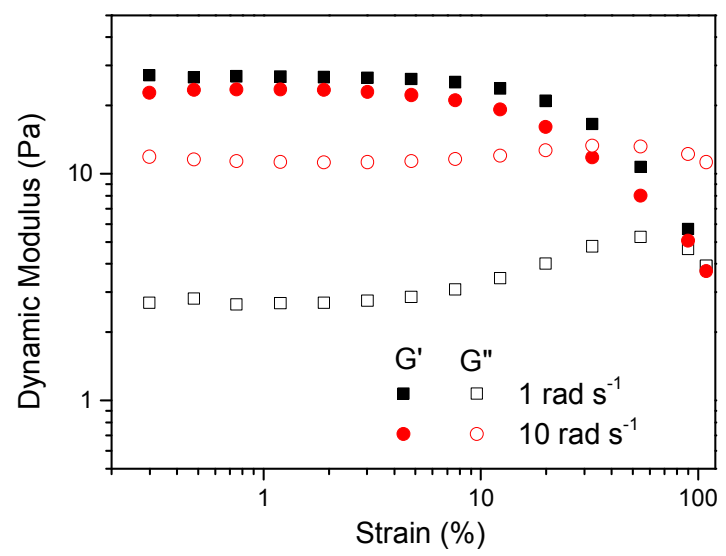

Figure S5 Dynamic moduli G' and G' for a typical HIPE hydrogel as a function of strain. 